\title{
A influência do lean healthcare para gestão hospitalar
}

\author{
Lizandra Garcia Lupi Vergara ${ }^{1}$ \\ Marcos Lucas de Oliveira ${ }^{2}$
}

\begin{abstract}
Resumo: O estudo da ergonomia envolve a verificação dos fatores que influenciam no desempenho do sistema produtivo e causam consequências aos trabalhadores. Dessa forma, procuram reduzir a fadiga, estresse, erros e acidentes proporcionando saúde, segurança e satisfação aos empregados, durante a sua interação com o sistema produtivo. Esse estudo objetivou identificar quais são as variáveis que intervém nos processos de operacionalização de sistemas complexos que utilizam os princípios da filosofia lean healthcare. A metodologia utilizada foi de natureza aplicada, com o uso de entrevistas focalizadas por meio de questionários. Os resultados do estudo apontam que os hospitais investigados possuem uma baixa implementação da filosofia lean e não há uma aferição da implementação com o aumento do nível de segurança laboral. Conclui-se que, de modo geral, a gestão hospitalar ainda está com cerne nos procedimentos operacionais e não com foco no paciente. Ademais, evidencia-se que os avanços no elo da gestão hospitalar no setor público, no sul do Brasil, são baixos e os métodos de gestão são, portanto, classificados como tradicionais e/ou obsoletos.
\end{abstract}

Palavras-chave: Ergonomia. Engenharia de resiliência. Sistemas complexos. Hospitais públicos. Lean healthcare.

\section{The influence of lean healthcare for hospital management}

Abstract: The study of ergonomics involves the verification of the factors that influence the performance of the productive system and cause consequences to the workers. In this way, they seek to reduce fatigue, stress, errors and accidents by providing health, safety and satisfaction to employees during their interaction with the production system. This study aimed to identify which are the variables that intervene in the processes of operationalization of complex systems that use the principles of the lean healthcare philosophy. The methodology used was of an applied nature, with the use of focused interviews through questionnaires. The results of the study indicate that the hospitals investigated have a low implementation of the lean philosophy and there is a measurement of the implementation with the increase of the level of job security. It is concluded that, in general, hospital management is still at the heart of operational procedures and not focused on the patient. In addition, it is evident that the advances in the hospital management link in the public sector in southern Brazil are low and management methods are therefore classified as traditional and / or obsolete.

Keywords: Ergonomics. Resilience engineering. Complex systems. Public hospitals. Lean healthcare.

\footnotetext{
${ }^{1}$ Doutorado em Ergonomia/Engenharia da Produção pela Universidade Federal de Santa Catarina (UFSC). Professora do programa de pós-graduação em Engenharia da Produção e Arquitetura pela Universidade Federal de Santa Catarina (UFSC). l.vergara@ufsc.br.

2 Doutorando em Engenharia da Produção pela Universidade Federal de Santa Catarina (UFSC). eng.marcos.lucas@gmail.com.
} 


\title{
La influencia de lean healthcare para la gestión hospitalaria
}

\begin{abstract}
Resumen: El estudio de la ergonomía implica la verificación de los factores que influyen en el desempeño del sistema productivo y causan consecuencias para los trabajadores. De esta manera, buscan reducir la fatiga, el estrés, los errores y los accidentes proporcionando salud, seguridad y satisfacción a los empleados durante su interacción con el sistema de producción. Este estudio tuvo como objetivo identificar cuáles son las variables que intervienen en los procesos de operacionalización de sistemas complejos que utilizan los principios de la filosofía de salud esbelta. La metodología utilizada fue de naturaleza aplicada, con el uso de entrevistas focalizadas a través de cuestionarios. Los resultados del estudio indican que los hospitales investigados tienen una baja implementación de la filosofía lean y hay una medición de la implementación con el aumento del nivel de seguridad laboral. Se concluye que, en general, la gestión del hospital sigue siendo el núcleo de los procedimientos operativos y no se centra en el paciente. Además, es evidente que los avances en el vínculo de gestión hospitalaria en el sector público en el sur de Brasil son bajos y, por lo tanto, los métodos de gestión se clasifican como tradicionales u obsoletos.
\end{abstract}

Palabras clave: Ergonomía. Ingeniería de resiliencia. Sistemas complejos. Hospitales públicos. Lean healthcare.

\section{Introdução}

A área da saúde corresponde a uma rede de organizações que prestam serviços a comunidade e atendem aos requisitos de órgãos regulatórios e governamentais (SOLIMAN e SAURIN, 2017b). Nesse contexto, em vista da imprevisibilidade e da variabilidade das atividades trabalhistas do setor da saúde (DE SOUZA e PIDD, 2011), os processos podem ser considerados complexos, dinâmicos e estocásticos (CAGLIANO, GRIMALDI e RAFELE, 2011). Para Azadeh, Roudi e Salehi (2017), atividades trabalhistas em sistemas complexos, estão propensas aos acidentes de trabalho de índices críticos. Montero (2013), Azadeh e Zarrin (2016), apontam que a decorrência desses são frutos da inexistência de um plano de gestão direcionado a prevenção.

Sobre essa análise, a engenharia de resiliência tendo como finalidade modificar e monitorar os modelos de gestão de riscos (DINH et al., 2012), procura métodos para aumentar a capacidade de criar processos robustos e flexíveis em todos os níveis de uma organização (AZADEH e ZARRIN, 2016). Conforme Azadeh et al. (2015) a engenharia de resiliência envolve os fatores humanos, a teoria do controle e a engenharia de segurança, com foco na eliminação dos processos ineficientes e/ou inseguros. Na posição de Furniss et al. (2011), a engenharia de resiliência maximiza as operações de maneira controlada, tanto em situações esperadas quanto em imprevisíveis, ou seja, oferece uma nova maneira de pensar sobre segurança e acidentes. 
Em consonância a descrição a priori, a filosofia lean healthcare é uma abordagem enxuta de gestão em saúde que consiste em agregar valor por meio do envolvimento de toda equipe na tomada das decisões tendo como foco o desenvolvimento de processos padronizados e confiáveis (HOUCHENS e KIM, 2014). Poksinska (2010) ressalva, no entanto, que a implementação da filosofia enxuta na área da saúde requer adaptação e desenvolvimento ao contexto específico da aplicação, com vistas a permitir que a equipe de saúde se aproprie da abordagem.

Na visão de Mintzberg (2012), a aplicação da filosofia lean healthcare vem sendo disseminada, em diversos países, desde o início dos anos 2000, através de pesquisas e compilações de diferentes casos, tópicos e abordagens nas unidades de saúde. A sua utilização no Brasil é considerada recente (HOUCHENS e KIM, 2014). Nesse âmbito, a filosofia lean atrai muitos pesquisadores que fundamentam o seu uso e exploração de seus conceitos da manufatura enxuta para o campo da saúde (RADNOR, 2011). Esses estudos, contudo, evidenciam que a concentração das aplicações do lean está direcionada as suas ferramentas em áreas ou processos específicos, e não diretamente como a cultura da organização (HAENKE e STICHLER, 2015; SOLIMAN e SAURIN, 2017b).

Nesse contexto, para Young et al. (2004) a filosofia lean healthcare apoia-se no conceito de produção como uma interação complexa de atividades individuais, isto é, visa melhorar o desempenho de uma organização de saúde por meio da identificação e consequentemente eliminação dos desperdícios e dos processos ineficazes dos sistemas de trabalho sócio-técnicos. Segundo Hendrick (1991), a teoria dos sistemas sócio-técnicos procura decodificar como funciona a organização das empresas e seus subsistemas, que se inter-relacionam e influenciam entre si (HENDRICK, 2008).

Perante a isso, Wilson (2014) e Holden et al. (2015) declaram que com o objetivo de transparecer o vínculo do atendimento as necessidades aos fatores e interações dos sistemas de trabalho sociotécnicos, os princípios fundamentais das condições humanas e da macroergonomia começaram a ser difundidos. Sobre essa ótica, Saurin (2014) aponta que na ergonomia a perspectiva da complexidade começou a ganhar força por meio do carregamento de informações confiáveis nos sistemas e com o uso de tecnologias inovadoras.

Diante disso, esse estudo buscou responder ao seguinte questionamento: A aplicação do lean healthcare é dependente do contexto? Além disso, objetivou identificar quais são as variáveis que intervém nos processos de operacionalização de sistemas 
complexos que utilizam os princípios da filosofia lean healthcare. Desse modo, por se tratar de um conhecimento multifacetário, isto é, que não se obtém de forma exata, utilizou-se na metodologia da aplicação de questionários semiestruturados e análise de conteúdo. Espera-se, enquanto contribuição teórica, que este estudo aumente a compreensão e também o debate sobre a filosofia lean healthcare.

\section{Fundamentação Teórica}

Nessa são apresentados os conceitos que relacionam-se com o objetivo desse estudo. Dessa forma, descritivamente são apresentados alguns conceitos, definições e abordagens da ergonomia, da engenharia de resiliência, dos sistemas complexos e do lean healthcare.

\subsection{Sistemas complexos e abordagem sócio-técnica}

A terminologia "complexidade" até o momento não possuí uma definição consensual, embora seja amplamente utilizada por diversos pesquisadores (JACOBSON et al, 2017). Para Érdi (2008), os sistemas complexos podem ser entendidos como interfaces que compartilham alguns atributos de inter-relação, podendo ser com alto número de elementos (JOHNSON, 2010), propriedades emergentes (ÉRDI, 2008), dinâmicas e não lineares (SNOWDEN e BOONE, 2007), e, de comportamentos adaptativos (KURTZ; SNOWDEN, 2003).

Na posição de Domingues, Batista e Tato (2017) os sistemas complexos podem ser entendidos como um instrumento de mudança e resiliência, ou seja, trata-se de um método com princípios e objetivos que visam maximizar as funções e eliminar as atividades desnecessárias. Por sua vez, Andrade et al. (2006) relatam que teoria dos sistemas sóciotécnicos procura decodificar como funciona a inter-relação das organizações e de seus subsistemas.

Dessa forma, para se obter efetividade nos resultados, a análise deve envolver todos os empregados e as mudanças devem fazer parte da cultura da empresa (OLIVEIRA, JORDÃO e VERGARA, 2019). Nesse sentido, Andrade et al. (2006), descreve que os subsistemas a ser considerados na teoria sócio-técnico são: (i) social; (ii) tecnológico; (iii) organizacional, e (iv) o ambiente externo. Hettinger et al. (2015), relatam que os estudos 
de sistemas sócio-técnicos complexos são uma classe particular dos sistemas adaptativos compostos por pessoas e tecnologias que interagem para produzir uma saída desejável (TRIST, 2017).

Wan et al. (2017), advertem que em um sistema sócio-técnico o termo social centra-se na relação entre as pessoas e seus atributos, como atitude, valor e habilidades, enquanto o termo técnico enfatiza os processos, tarefas e tecnologias para produzir o resultado designado. Nessa perspectiva, a organização passa a ser mapeada em dois subsistemas: (i) subsistema técnico, que são máquinas, técnicas, entre outros e (ii) subsistema social, que são os indivíduos e grupos de empregados com seus comportamentos, culturas, capacidades, sentimentos, entre outros (WAN et al., 2017).

Nesse sentido Soliman e Saurin (2017a), revelam que os sistemas sócio-técnicos complexos não podem ser modelados matematicamente, pois os atributos avaliados possuem comportamento instáveis. Desse modo, a abordagem dos sistemas sócio-técnicos, a partir de uma visão crítica realista, pressupõe a existência de uma realidade objetiva (SOLIMAN e SAURIN, 2017a). Assim, passa a ser possível a observação da subjetividade do indivíduo, e a possibilidade do sistema complexo ser medido (WALKER et al., 2010). Em outras palavras, há uma possibilidade de identificar a complexidade das tarefas, em um mesmo ambiente de trabalho, entre mais complexa e menos complexa.

Assim, para Dekker et al. (2013) a perspectiva do realismo crítico é relevante para o gerenciamento das operações de sistemas complexos, uma vez que há a possibilidade de reconhecer e conceber práticas para influenciar os fatores e suas interações que estabelecem o cenário para a emergência da complexidade. Concomitantemente Pavard e Dudgale (2002), apontam que na perspectiva sócio-técnica uma organização interage com o ambiente sendo capaz de autorregulação e equifinalidade. Isto é, pode alcançar um mesmo objetivo a partir de diferentes caminhos e por meio da utilização de diferentes recursos.

No entender dos autores Wan et al. (2017), os sistemas sócio-técnicos são introduzidos nas organizações como meio de compreender as perspectivas sociais e tecnológicas, esses dois conjuntos afetam intrinsicamente a produção de bens e serviços. É nesse contexto, que a engenharia de resiliência se insere no âmbito da saúde, pois ela usa de insights de pesquisa sobre falhas em sistemas complexos, incluindo contribuintes organizacionais para o risco e os fatores que afetam o desempenho humano, para gerenciá- 
los de forma proativa por meio de ferramentas de engenharia (RIGHI, SAURIN e WACHS, 2015).

\subsection{Lean healthcare}

Nas últimas décadas, o setor de assistência médica avançou na adaptação dos princípios do Lean nas operações de assistência médica, embora a maneira e os resultados encontrados tenham variado conforme a característica da unidade de saúde (RADNOR, 2011). As organizações de saúde estão implementando o Lean por meio de pequenos projetos fechados que estão criando bolsões de melhores práticas (SOUZA e SOUZA, 2009).

A revisão da literatura mostra que houve alguns resultados tangíveis significativos nas organizações de saúde que adotaram princípios Lean, como aumento do rendimento do paciente (DICKSON et al., 2008), redução do tempo de espera (AL-ARAIDAH et al., 2010) e melhorias no ambiente de trabalho (KAPLAN e PATTERSON, 2008). No entanto, outros autores identificaram barreiras ao implementar a gestão Lean (MINTZBERG, 2012), em organizações de saúde como, por exemplo, a falta de propriedade dos processos propostos, ceticismo e resistência à mudança (SOUZA e PIDD, 2011).

Conforme o estudo de Soliman e Saurin (2017b) as barreiras culturais e práticas devem ser superadas para uma correta e eficaz disseminação da aplicação da filosofia lean no setor da saúde. Kim et al. (2006) e D'andreamatteo et al. (2015) afirmaram que a prontidão, por parte de profissionais técnicos em saúde, com a mudança cultural, tem fator positivo e decisivo na implementação do lean healthcare na unidades de saúde.

\subsection{Sistema Lean e a engenharia de resiliência}

A engenharia de resiliência procura maneiras de melhorar o desempenho das organizações em uma variedade de condições, a fim de evitar resultados indesejáveis (RENIERS; SORENSEN e KHAN, 2014). Este campo da engenharia traz conceitos baseados principalmente em competências relacionadas à capacidade de detecção, antecipação e prevenção (AZADEH; ROUDI e SALEHI, 2017).

A aplicação dos conceitos de engenharia de resiliência é vista como essencial para o desenvolvimento de um sistema de gestão eficaz, isto é, com capacidades flexíveis, e, ao 
mesmo tempo robustas, de adaptação a diferentes situações de intervenção, monitoramento e antecipação de riscos (FERNANDES; HURTADO e BATIZ, 2015). Para Righi, Saurin e Wachs (2015), a engenharia de resiliência pode ser projetada para sistemas sócio-técnicos complexos de modo a suportar o uso da capacidade de adaptação.

De acordo com os esses autores, a engenharia de resiliência utiliza de três critérios para a avaliação de teorias de gerenciamento de segurança, esses são: (i) foco de aplicação, nível do sistema abordado pela teoria; (ii) poder da teoria, extensão em que a teoria permite previsibilidade e controle do sistema e (iii) disponibilidade tecnológica, medida em que a teoria foi testada e avaliada (RIGHI; SAURIN; WACHS, 2015).

Seguindo essa linha de pensamento Hollnagel (2011) define quatro habilidades que caracterizam os sistemas resilientes: (i) antecipar; (ii) monitorar; (iii) responder; e (iv) aprender. Além disso, Back et al. (2008) relatam que a engenheira de resiliência deve ser aplicada em três níveis: (i) individual, (ii) equipe e (iii) organizacional.

Caso haja um subtítulo, seguir sequencia com numeração correspondente à fundamentação, ex: 2.1 escreva o subtítulo; usar letra Times New Roman ou Arial, tamanho 12, espaçamento 1,5 entre linhas, com entrada de parágrafo de 1,25 (alínea).

\section{Procedimentos Metodológicos}

Essa sessão tem como propósito apresentar os instrumentos de coleta de dados que foram utilizados nessa pesquisa. Além disso, descrevem as etapas percorridas para atingir o objetivo do estudo.

\subsection{Delineamentos da pesquisa}

O artigo é de natureza aplicada porque objetivou verificar quais são as variáveis que intervém nos processos de operacionalização de sistemas complexos que utilizam os princípios da filosofia lean healthcare (GIL, 2010). Em relação ao método científico, a pesquisa é caracterizada como indutiva porque teve por objetivo levar a conclusões, cujo conteúdo é mais amplo do que das premissas que se baseou (MARCONI e LAKATOS, 2010). 
A abordagem do estudo é classificada como qualitativa (MIGUEL, 2010), porque tem ênfase na obtenção de informações sobre a utilização da filosofia lean healthcare em três hospitais escola do sul do Brasil. Assim, através da análise de conteúdo foi possível obter as identificações de conteúdos que foram codificados em categorias de resposta (BARDIN,1994). Para Boni e Quaresma (2015), esse método apresenta como vantagem a flexibilidade de interação entre o entrevistado e o entrevistador, em vista que favorece a compilação de respostas espontâneas.

Em referência ao objetivo da pesquisa, esse artigo pode ser considerado exploratório (GIL, 2010), porque se buscou um aprofundamento da temática, sobre a ótica dos participantes, em prol do aprimoramento da literatura científica. Quanto aos procedimentos técnicos, utilizou-se de uma pesquisa bibliográfica para gerar $\mathrm{o}$ embasamento teórico do estudo. Para Marconi e Lakatos (2010), baseia-se em materiais que ainda não receberam um tratamento analítico e que podem ser reelaborados de acordo com os objetivos da pesquisa.

\subsection{Delimitações da pesquisa}

Essa pesquisa tem como cerne três hospitais escola que atuam com a filosofia lean healthcare na região sul do Brasil. A escolha por essa classe de hospitais ocorreu por meio dos fatores de contexto de interesse a comunidade acadêmica, visto que esses hospitais representam as divisões distintas e reconhecidas no domínio do Sistema Único de Saúde (SUS) na região.

Nessa pesquisa fazem parte do aspecto amostral os profissionais e responsáveis técnicos que são responsáveis pelo desenvolvimento da filosofia lean healthcare em alguma unidade e/ou setor do hospital investigado. Assim, não compuseram a pesquisa os demais profissionais de saúde que não estão vinculados no planejamento e desenvolvimento da filosofia lean na unidade hospitalar analisada.

\subsection{Instrumentos de coleta e análise dos dados}

Para atingir os objetivos do presente estudo e responder a problemática da pesquisa, utilizou-se de um procedimento sequencial de atividades. A Figura 2 apresenta as etapas e processos seguidos para obtenção dos resultados. 


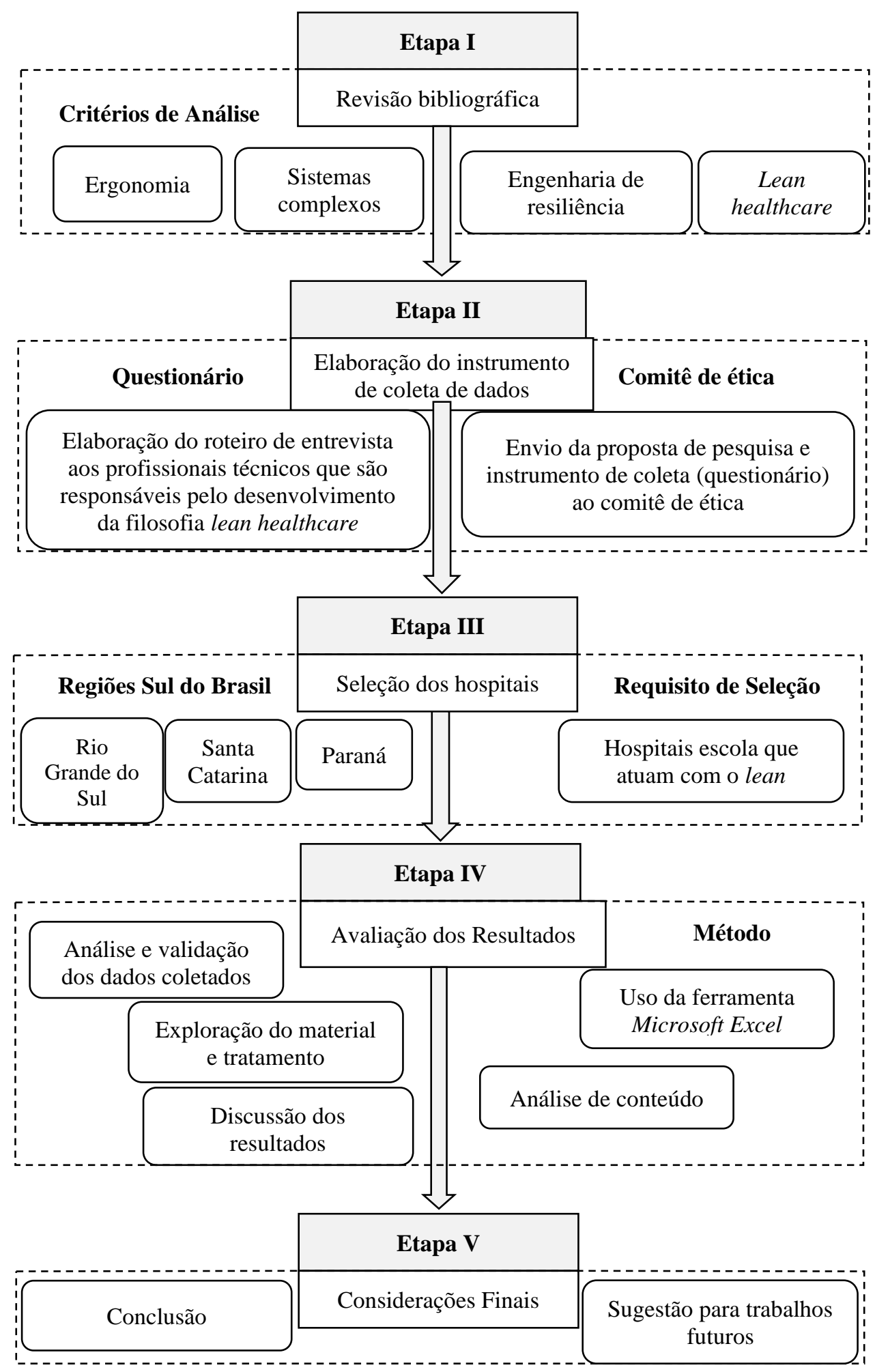

Figura 2 - Estrutura da pesquisa 
Para o desenvolvimento da abordagem metodológica desse estudo foi garantido ao entrevistado à liberdade do aceite ou não em participar dessa pesquisa. Diante disso, somente após o aceite e consentimento dos entrevistados que se prosseguiu com a coleta dos dados.

\subsubsection{Instrumento de coleta de dados}

O instrumento de coleta de dados utilizado refere-se à aplicação de questionários semiestruturado in loco. Esse teve como objetivo levantar dados sobre a compreensão dos responsáveis técnicos sobre a filosofia lean healthcare. Nesse sentido, no Quadro 1 apresenta o roteiro seguido para as entrevistas.

Quadro 1: Roteiro das entrevistas

\begin{tabular}{|c|l|}
\hline $\mathbf{N}^{\mathbf{0}}$ & \multicolumn{1}{|c|}{ Questionamento } \\
\hline 1 & Qual o seu nome? \\
\hline 2 & Qual a sua idade? \\
\hline 3 & Qual a sua formação base? \\
\hline 4 & Qual cargo e/ou função você exerce na empresa? \\
\hline 5 & Há quanto tempo você atua com o lean healthcare? \\
\hline 6 & Em sua opinião, quais são as dificuldades de implantação no lean healthcare? \\
\hline 7 & Em sua opinião, o que a aplicação do lean healtcare trouxe para a organização? \\
\hline 8 & Em sua opinião, os princípios lean são compreendidos pelos profissionais da área da saúde? \\
\hline 9 & $\begin{array}{l}\text { Em sua opinião, quais são os desafios e/ou barreiras que a filosofia lean enfrenta por parte dos } \\
\text { empregados na área da saúde? }\end{array}$ \\
\hline 10 & $\begin{array}{l}\text { De acordo com as suas experiências, quais foram às ações após inserção da filosofia lean healthcare } \\
\text { para a segurança hospitalar? }\end{array}$ \\
\hline
\end{tabular}

\section{Fonte: Autores}

Aplicação do questionário aos entrevistados visou também levantar informações sobre a influência da filosofia com a gestão da segurança hospitalar. Assim, a aplicação desse instrumento pode conduzir a discussões que estabelecem perguntas adicionais, sem deixar de seguir com o objetivo da entrevista, mas sim de melhorar o direcionamento das respostas dos entrevistados.

O levantamento de dados foi realizado por meio de entrevista semiestruturadas com 15 funcionários distribuídos entre as diversas áreas do hospital escola. As entrevistas foram realizadas entre os meses de junho e setembro, sendo as falas dos entrevistados gravadas, essa com média de duração média de até 5 minutos. Tais verbalizações foram transcritas e 
analisadas por meio da análise do conteúdo, que consiste em um conjunto de técnicas de análise das comunicações que utiliza procedimentos sistemáticos e objetivos de descrição do conteúdo (BARDIN, 1994).

A utilização do método, nesse artigo, foi às inferências de conhecimento dos entrevistados relativas à filosofia lean healthcare e a gestão da segurança hospitalar. Nesse contexto, a partir da transcrição e análise das entrevistas ocorreu a ordenadação das palavras chave em prol do agrupamento das possíveis respostas em categorias. Essa etapa visou propiciar rigor aos dados e fornecer uma objetividade quanto às perguntas versus respostas declaradas pelos entrevistados. De forma sistemática, a análise de conteúdo utilizada nesse artigo foi separada em três etapas, como evidencia o Quadro 2.

Quadro 2: Etapas da análise de conteúdo

\begin{tabular}{|c|l|}
\hline Etapa & \multicolumn{1}{c|}{ Realização } \\
\hline Pré- análise & $\begin{array}{l}\text { Realizou-se a operacionalização e sistematização das ideias, ou seja, a leitura fluente } \\
\text { das entrevistas. }\end{array}$ \\
\hline $\begin{array}{c}\text { Exploração do } \\
\text { material }\end{array}$ & $\begin{array}{l}\text { O conteúdo foi codificado, categorizado e quantificado, isto é, realizou-se a } \\
\text { separação das temáticas e de suas problemáticas. A análise ocorreu por meio de duas } \\
\text { tipologias: (i) semelhança, as problemáticas foram agrupadas em categorias e } \\
\text { subcategorias, e, (ii) categorial, os fatores explicativos, da mesma entrevista, não } \\
\text { foram enquadrados em apenas uma categoria, em vista das respostas corresponderem } \\
\text { a todas as variáveis investigadas. }\end{array}$ \\
\hline $\begin{array}{c}\text { Tratamento dos } \\
\text { resultados }\end{array}$ & Os dados foram tratados quantitativamente por meio de uma interpretação qualitativa. \\
\hline
\end{tabular}

Fonte: Autores com base em Bardin (1994).

Por meio do Quadro 2 e da Figura 3 é possível visualizar de forma sintetizada a etapa de exploração do material, quanto às tipologias, isto é, semelhança e categorial. Diante disso, com vistas a não identificar os entrevistados e o local de atuação dos mesmos, para a compilação dos resultados, utilizou-se de uma representação técnica $\left(\operatorname{En}^{\circ}\right)$.

Essa representação, teoricamente, representa as entrevistas e as descrições dos 15 profissionais entrevistados, que na etapa de resultados são representados tecnicamente como E1 até E15. Em consonância ao descrito, foi resguardado a idoneidade das respostas dos entrevistados. 
Figura 3 - Visualização sintetizada da etapa de exploração do material

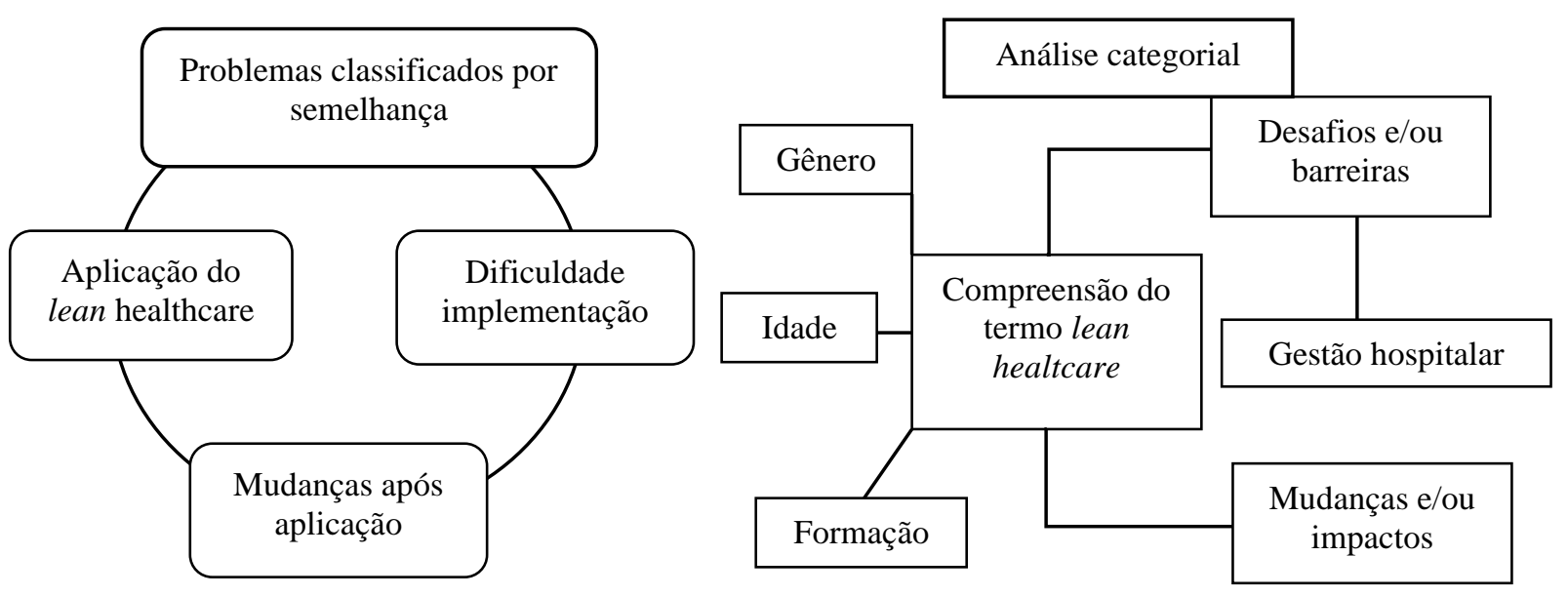

Fonte: Autores

A análise categorial referiu-se, mais especificamente, a uma análise abstrata e não exclusiva, em vista de em uma mesma entrevista decorrer fatores explicativos que não se enquadraram apenas em uma categoria (BARDIN, 1997). Posto isto, a representação esquemática construída apresenta as variáveis que incidiram sobre a aplicação da filosofia lean, nos ambientes hospitalares pesquisados. Desse modo, finalizada a etapa de exploração do material iniciou-se o tratamento dos resultados, que é apresentado na próxima seção desse artigo.

\section{Descrição e Análise dos Resultados}

Nessa etapa do estudo têm-se os relatos e as suas percepções quanto à multifuncionalidade da filosofia lean healthcare e sua inferência na segurança dos sistemas hospitalares pelos gestores e/ou profissionais técnicos que são responsáveis pelo desenvolvimento da filosofia lean na unidade hospitalar. Dessa forma, os relatos coletados tiveram como objetivo ilustrar e/ou gerar hipóteses sobre o tema pesquisado.

Desse modo, por meio das primeiras perguntas aos entrevistados, como formação base e função que exerce na empresa, pode-se evidenciar que os facilitadores da filosofia lean, nas unidades hospitalares investigadas, possuem formações multidisciplinares. Sendo evidenciadas, principalmente, as profissões de técnico administrativo, psicólogo, enfermeiros e médicos. 
Concomitante a essa declaração, todos os quinze entrevistados declararam conhecer os cinco princípios da filosofia lean (Quadro 3). Além disso, percebeu-se que a filosofia lean healthcare nos hospitais investigados está em fase inicial de implementação.

Quadro 3: Princípios lean em serviços

\begin{tabular}{|c|c|c|}
\hline $\mathbf{N}^{\mathbf{0}}$ & Princípio & Característica \\
\hline \multirow[t]{2}{*}{1} & Especificar valor & $\begin{array}{l}\text { Identificar o que o cliente pondera como valor, isto é, pelo que está disposto } \\
\text { a pagar. }\end{array}$ \\
\hline & $\begin{array}{l}\text { Identificar cadeia } \\
\text { de valor }\end{array}$ & $\begin{array}{l}\text { Identificar quais as etapas agregam valor, isto é, eliminar os desperdícios. } \\
\text { Essa etapa é executada por meio do mapeamento de fluxo de valor. }\end{array}$ \\
\hline \multirow[t]{2}{*}{3} & $\begin{array}{l}\text { Criar fluxo } \\
\text { contínuo }\end{array}$ & Visa atender as necessidades dos clientes com agilidade e eficiência. \\
\hline & $\begin{array}{l}\text { Introduzir a } \\
\text { produção puxada }\end{array}$ & $\begin{array}{l}\text { Deixar o cliente "puxar" o produto, isto é, objetiva atender a demanda no } \\
\text { momento necessário, na quantidade necessária e no tempo necessário. }\end{array}$ \\
\hline${ }_{4} \zeta$ & $\begin{array}{l}\text { Introduzir a } \\
\text { melhoria contínua }\end{array}$ & $\begin{array}{l}\text { Engajamento dos colaboradores na proposição de melhorias e autoavaliação } \\
\text { dos processos. }\end{array}$ \\
\hline
\end{tabular}

Fonte: Autores com base em Pinto (2009).

As alas hospitalares que possuem maiores índices de implementação, conforme descrição dos entrevistados, são: (i) farmácia hospitalar, visa à otimização do estoque e controle de licitações, entradas e saídas; e, (ii) hospedaria, visa a ordenação dos leitos quanto a preparação de pacientes que estão em tramites de desocupar o quarto "dar alta" versus setor de higienização ou ainda do inverso, isto é, do tempo de higienização versos a disponibilidade do leito para entrada do paciente "dar baixa".

Conforme Wan et al. (2017) em um sistema sócio-técnico o comportamento das pessoas, frente ao trabalho, é fielmente dependente da organização deste trabalho e do conteúdo de tarefas a ser executadas pelos mesmos. Ao sistema de prestação de serviços hospitalares Mans e Van (2015) relatam que há uma relação de dependência da execução adequada do trabalho e da eficácia aos processos. Nesse sentido, a entrevistada E7 descreveu que:

O processo de trabalho de uma unidade hospitalar é apoiado por atividades clínicas e não clínicas, executadas por diferentes tipos de recursos (médicos, enfermeiros, especialistas técnicos, dentistas, balconistas) e podem variar de uma organização para outra.

Conforme Rojas et al. (2016), os processos de cuidados de saúde são uma série de atividades destinadas a diagnosticar, tratar e prevenir qualquer doença, a fim de melhorar a saúde de um paciente. Nesse sentido, uma das categorias decorrente das entrevistas fez 
referência às dificuldades de implantação da filosofia lean healthcare, assim o Quadro 3 aponta os resultados encontrados a essa categoria.

Quadro 3: Categoria dificuldades de implementação

\begin{tabular}{|c|l|c|}
\hline Categoria & \multicolumn{1}{|c|}{ Definição } & Entrevistado (s) \\
\hline $\begin{array}{l}\text { Dificuldades de } \\
\text { implementação }\end{array}$ & * falta de capacitação & \\
& $*$ terceirizados & * visão sistêmica \\
& $*$ característica do trabalho & E1, E2, E3, E4, E5, E8, E9, \\
\end{tabular}

Fonte: Autores

Os demais entrevistados na categoria dificuldade de implementação, responderam não conhecerem quais são as dificuldades de implementação do lean healthcare, porque consideraram estarem em fase inicial de planejamento para introdução da filosofia, ou seja, não possuíam as compreensões necessárias a sua implementação identificadas. Nesse contexto, Rouch et al. (2011) declara que a filosofia lean healthcare representa um sistema de gerenciamento dos sistemas de saúde hospitalar que tem como objetivo otimizar o atendimento por meio da identificação e eliminação dos desperdícios.

Os principais objetivos do lean healthcare, segundo o autor Rouch et al. (2011), podem ser identificados por seis etapas, sendo essas: (i) otimização dos processos e tempo de espera do paciente; (ii) padronização e otimização das interfaces entre as diferentes divisões e enfermarias; (iii) integração dos sistemas de informações; (iv) redução dos retrabalhos; (v) minimização de estoques, e (vi) redução do tempo de permanência dos pacientes na unidade hospitalar (WELLMAN; HAGAN e JEFFRIES, 2011).

Para o entrevistado E8 toda mudança radical em um ambiente de trabalho causa certa resistência por parte dos trabalhadores, em vista da falta de reconhecimento da necessidade de alteração da forma como a atividade é executada. E9 complementa relatando que os colaboradores precisam evidenciar seus desperdícios e os gargalos nos quais estão acostumados a conviver em suas atividades diárias. Em consonância com os demais entrevistados E1 cita que:

A aceitação da filosofia lean depende do comportamento e apoio da liderança, ou seja, os lideres precisam ser os primeiros demonstrarem as práticas do pensamento lean na organização.

Segundo a pesquisa dos autores Al-Balushi et al. (2014), há sete "fatores de prontidão" que devem ser desenvolvidos pela gestão dos hospitais antes da introdução da 
filosofia lean no ambiente laboral, sendo esses: (i) apoio da equipe de liderança; (ii) alinhamento estratégico; (iii) identificação dos valores e clientes; (iv) identificação dos processos e eliminação dos desperdícios; (v) treinamento e envolvimento das pessoas a filosofia lean; (vi) desenvolvimento de métricas de avaliação de desempenho e recompensas alinhado aos objetivos Lean; e (vii) nivelamento da demanda e da capacidade para melhorar o fluxo. Nesse contexto, o entrevistado E2 argumenta que:

As atividades diárias de um hospital não são concisas com o pensamento de maximização de eficiência, porque trabalhamos com altas variações de demandas, isto é, tratamos muitos pacientes simultaneamente.

Dentre esses fatos, um dos fatores a ser considerado dentro dos hospitais públicos, principalmente, aos hospitais-escola é o atendimento das expectativas dos pacientes quanto à prestação de serviço (MOLDOVAN, 2018), ou seja, como a entidade pública gerencia seus recursos em vista das altas demandas. Nesse contexto, a filosofia lean healthcare pode auxiliar por meio da identificação dos elos de trabalho que geram valor e eliminar as que são dispendiosas (D’ANDREAMATTEO et al., 2015). Sobre essas argumentações no Quadro 4 são expressos os resultados encontrados para a categoria "aplicação do lean healthcare" nos hospitais investigados.

Quadro 4: Categoria aplicação do lean healthcare

\begin{tabular}{|c|l|c|}
\hline Categoria & \multicolumn{1}{|c|}{ Definição } & \multicolumn{1}{|c|}{ Entrevistado (s) } \\
\hline $\begin{array}{c}\text { Aplicação do lean } \\
\text { healthcare }\end{array}$ & $\begin{array}{l}\text { * aumento da segurança do paciente } \\
\text { * redução de custos } \\
\text { *redução de erros } \\
\text { *melhorias de layouts }\end{array}$ & E2, E5, E7, E10, E12 e E15. \\
\hline
\end{tabular}

Fonte: Autores

$\mathrm{Na}$ opinião do entrevistado E5 o facilitador da filosofia lean precisa observar de forma direta o funcionamento da organização para orientar os funcionários na identificação de atividades que agregam valor, principalmente no setor da saúde, pois nós não possuímos uma visão de gestão. Para E1, os princípios do lean não são facilmente aceitos pelos profissionais da área da saúde, porque não estão em uma linguagem simples, de cotidiano nosso cotidiano, o que impõem certa estranheza. Nessa conjuntura, Costa e Godinho Filho (2016) descrevem que a implementação do lean na área da saúde está sujeita a algumas limitações, tendo em vista o baixo índice de conhecimento das ferramentas e dos métodos de gestão existentes. O entrevistado E12 cita que: 


\begin{abstract}
A natureza do lean por si parece variar consideravelmente de um lugar para outro, desde a utilização de uma única ferramenta enxuta até a construção de uma cultura enxuta. Contudo, no hospital fica difícil passar essa filosofia uma vez que os profissionais da saúde não possuem a compreensão básica de gestão.
\end{abstract}

À medida que os líderes da área da assistência à saúde se familiarizam com o pensamento lean, eles constatam que um dos seus três princípios básicos é a insistência em manter um ambiente focado no cliente (E14). Nesse aspecto, os estudos de Radnor, Holweg e Waring (2012) relatam que é dada pouca atenção à definição de valor a partir da perspectiva do paciente, o que prospecta uma limitante da aplicação da filosofia.

Essa visão vai ao encontro do relato do entrevistado E7 que argumenta existir feudos profissionais na área hospitalar. Concomitantemente E3 argumenta que o processo hospitalar não é centrado no paciente e sim na atuação profissional dos médicos. Seguindo essa linha de raciocínio, E11 declara que uma das barreiras à implantação da filosofia lean são as próprias características dos hospitais escola, porque lidam com mais de uma classe de contratação de profissionais como, por exemplo, terceirizados e estatutários, além dos residentes e/ou demais estagiários.

Sobre outra visão, mas em consonância com os problemas de implementação do lean healthcare, o estudo de Henrique et al. (2016) faz uma crítica a forma como a implantação da filosofia lean é realizada nos sistemas de saúde, pois para esse autor as técnicas e diretrizes usadas atualmente, para gerir um sistema de saúde enxuto, são simples adaptações do modelo original da produção enxuta (lean production), que foi inicialmente direcionado para a manufatura.

$\mathrm{Na}$ execução de um fechamento ao assunto, a pesquisa de Henrique et al (2016) declara que ainda não há uma consideração das atividades de suporte de forma representativa ao fluxo do paciente na atual conjuntura da aplicação do lean healthcare, ou seja, não são consideradas as informações do uso dos materiais versus o fluxo dos pacientes envolvidos na transformação de um paciente doente em um paciente saudável. No Quadro 6, são apresentados os resultados encontrados para a categoria “Ações para segurança hospitalar".

Quadro 6: Categoria ações para segurança hospitalar 


\begin{tabular}{|c|l|c|}
\hline Categoria & \multicolumn{1}{|c|}{ Definição } & Entrevistado (s) \\
\hline & * melhorias nas informações & \\
Ações para & *redução de tempo de espera & * redução de retrabalhos \\
segurança hospitalar & *eficácia & E1, E2, E3, E4, E5, E7, E8, \\
& *redução de custos & E9, E11, E13, E15 \\
& *Satisfação do clima organizacional & \\
\hline
\end{tabular}

Fonte: Autores

Para o entrevistado E10 a filosofia lean auxilia a assistência médica a lidar de forma mais humana com os pacientes. Essa declaração revela em si a característica central do lean healthcare. De acordo com Ker et al. (2014), a avaliação da implementação do lean healthcare tem por objetivo repassar as informações a alta gerência da instituição de saúde. Além disso, visa questionar a equipe de implementação enxuta sobre a relação entre a quantidade de recursos investidos e o nível de progressão alcançado (NARAYANAMURTHY e GURUMURTHY, 2018). Esses recursos podem ser: (i) horashomem; (ii) treinamento de mão-de-obra; (iii) necessidade de consultores externos; (iv) retrabalhos e (v) etc. Nesse contexto, o entrevistado E12 cita:

A melhoria dos processos de saúde pode ter um alto impacto na qualidade de vida dos pacientes. No entanto, aperfeiçoá-los não é uma tarefa fácil, pois vários desafios estão sempre presentes. Há sempre a necessidade de reduzir o custo dos serviços e melhorar as capacidades para atender à demanda, reduzir o tempo de espera do paciente, melhorar a produtividade dos recursos e aumentar a transparência dos processos.

Narayanamurthy e Gurumurthy (2018), destacam que na implementação da filosofia lean healtchare as instituições de saúde hesitam em avaliar principalmente os dados financeiros, isto é, o custo operacional da nova cultura e seu retorno imediato à entidade, ao invés de analisar os benefícios sociais, trabalhistas e organizacionais que o lean healtcare pode trazer para a organização. Na compreensão de Poksinska, FialkowskaFilipek e Engström (2016) são nos encontros entre médico-paciente que ocorre o principal elo para a criação de valor pela perspectiva do paciente, pois nesse momento que a qualidade funcional do cuidado é avaliada pelo cliente. 


\section{Discussão Dos Resultados}

Diante do exporto e das demais declarações dos entrevistados evidenciam-se que a aplicação do lean healthcare na região sul do Brasil encontra-se em fase inicial de implementação sujeita, portanto, a pequenas intervenções. Ademais, pode-se evidenciar que as principais dificuldades para implementação da filosofia lean na área hospitalar é a mudança de cultura e a baixa qualificação dos profissionais envolvidos em gestão na saúde.

Nesse sentido, sobre analise dos dados, quanto as palavras ou expressões que mais foram repetidas para as barreiras de implementação do lean healthcare tem-se: (i) resistência à mudança; (ii) variabilidade do processo; (iii) egos profissionais; e (iv) dificuldade para compreender valor e perdas. Em consonância ao descrito, pode-ser evidenciar por meio das entrevistas que a aplicação da filosofia lean healthcare necessita de melhores adaptações do lean manufacture, uma vez que precisa considerar as demais variações para sua devida adaptação aos cuidados de saúde, pois, a exemplo, não há uma consideração das atividades de suporte ao fluxo do paciente, na atual conjuntura da aplicação do lean healthcare.

Dessa forma, evidencia-se que a aplicação do lean healthcare é dependente do contexto e não há nenhum atalho para a compreensão de suas suposições subjacentes e princípios fundamentais. Por fim, declarasse que a consideração das outras variáveis ao lean healthcare poderá ser relevante para o aumento da eficiência de sua implementação nos hospitais, uma vez que passará a incluir os profissionais de saúde em prol da garantia da qualidade, segurança e de um serviço adequado ao usuário.

\section{Conclusões}

Este estudo abordou as percepções dos profissionais técnicos responsáveis pela execução da filosofia lean nos âmbitos hospitalares pesquisados. Nesse sentido, a análise dos resultados obtidos revela que aplicação do lean healthcare é dependente do contexto em que a filosofia será inserida. Como resultados desse estudo também evidenciam que uma das principais variáveis que intervém nos processos de operacionalização de sistemas complexos que utilizam os princípios da filosofia lean healthcare é a mudança de cultura já instaurada nas organizações hospitalares. 
Ademais, por meio de inferências de outros estudos pode-se apontar que uma das possíveis causas da baixa efetivação da filosofia é a falta de adaptação correta do lean manufacture a área da saúde. Dessa forma, de modo geral observa-se que os resultados encontrados nesse estudo vão ao encontro das atuais pesquisas descritas na literatura sobre o assunto. Portanto, conclui-se que, por mais que os hospitais escola do sul do Brasil estejam em fase iniciais da implementação do lean healthcare, há profissionais que conhecem a filosofia e reconhecem a sua aplicação para aprimorar os processos ineficazes na área da saúde.

Diante disso, para trabalhos futuros sugere-se a utilização de uma amostra mais representativa com o objetivo de se obter uma síntese mais aprofundada sobre a situação da filosofia lean healthcare no Brasil, e com vistas a extrair insights mais profundos sobre as adaptações da produção enxuta as operações de saúde. Por fim, declarasse que o presente trabalho foi realizado com apoio do Conselho Nacional de Desenvolvimento Científico e Tecnológico (CNPq) e da Universidade Federal de Santa Catariana (UFSC).

\section{Referências}

AL-ARAIDAH, O., et al. Lead-time reduction utilizing lean tools applied to healthcare: the inpatient pharmacy at a local hospital, Journal for Healthcare Quality, v. 32, p. 5966, 2010.

ANDRADE, A. L.; SELEME, A.; RODRIGUES, L. H.; SOUTO, R. Pensamento sistêmico: caderno de campo: o desafio da mudança sustentada nas organizações e na sociedade. Porto Alegre, Brasil: Bookman, 2006.

AZADEH, A. et al. A unique algorithm for the assessment and improvement of job satisfaction by resilience engineering : Hazardous labs. International Journal of Industrial Ergonomics, v. 49, p. 68-77, 2015.

AZADEH, A.; ZARRIN, M. An intelligent framework for productivity assessment and analysis of human resource from resilience engineering, motivational factors, HSE and ergonomics perspectives. Safety Science, v. 89, p. 55-71, 2016.

AZADEH, A; ROUDI, E.; SALEHI, V. Optimum design approach based on integrated macro-ergonomics and resilience engineering in a tile and ceramic factory. Safety Science, v. 96, p. 62-74, 2017.

BACK, J. et al. Resilience markers for safer Systems and organisations. Computer safety, reliability, and security, p. 99-112, 2008.

BARDIN, L. Análise de conteúdo. Lisboa: Edições 70, 1977. 
CAGLIANO, A. C.; GRIMALDI, S.; RAFELE, C. A systemic methodology for risk management in healthcare sector. Safety Science, v. 49 (5), p. 695-708, 2011.

COSTA, L. B. M.; GODINHO FILHO, M. Lean healthcare: review, classification and analysis of literature. Production Planning and Control, v. 27, n. 10, p. 823-836, 2016. D'ANDREAMATTEO, A. et al. Lean in Healthcare: a comprehensive review. Health Policy, v. 119, p.1197-1209, 2015.

DE SOUZA, L. B; PIDD, M. Exploring the barriers to lean health care implementation. Public Money \& Managemen, v. 31, n. 1, p. 59-66, 2011.

DEKKER, S. et al. Complicated, complex, and compliant: best practice in obstetrics. Cogn Technol Work, Applied Ergonomics, v. 15, p. 189-95, 2013.

DICKSON, E. W., ET. AL. Application of Lean Manufacturing Techniques in the Emergency Department, Journal of Emergency Medicine, v. 37(2), p.177-182, 2008.

DINH, L.T. et al. Resilience engineering of industrial processes: principles and contributing factors. Journal of Loss Prevention in the Process Industries, v. 25 (2), p. 233-241, 2012.

DOMINGUES, M.; BATISTA, A. L. F.; TATO, D. M. International Journal of Mining Science and Technology Engineering complex systems applied to risk management in the mining industry. International Journal of Mining Science and Technology, v. 27, n. 4, p. 611-616, 2017.

ÉRDI, P. Complexity explained. Berlin: Springer, 2008.

FERNANDES, P. R.; HURTADO, A. L. B.; BATIZ, E. C. Ergonomics management with a proactive focus. Procedia Manufacturing, v. 3, n. Ahfe, p. 4509-4516, 2015.

FURNISS, D., et al. A resilience markers framework for small teams. Reliability Engineering \& System Safety, v. 96 (1), p. 2-10, 2011.

GIL, A. C. Como Elaborar Projetos de Pesquisa. São Paulo. 5 Ed. Editora: Atlas. 2010.

HAENKE, R.; STICHLER, J.F.; Applying lean six sigma for innovative change to the post-anesthesia care unit. Journal of Nursing Administration, v. 45, n. 4, p. 185-187. 2015.

HENDRICK, H.W. Applying ergonomics to systems: Some documented "lessons learned". Applied Ergonomics, v. 39, n. 4, p. 418-426, 2008.

HENDRICK, H.W. Ergonomics in organizational design and management. Ergomics, v 34, n. 6, p. 743-756, 1991.

HETTINGER, L. J. et al. Modelling and simulation of complex sociotechnical systems: envisioning and analysing work environments. Ergonomics, v. 58, p. 600-14, 2015.

HOLDEN, R. J. et al. Healthcare workers' perceptions of lean: A context-sensitive, mixed methods study in three Swedish hospitals. Applied Ergonomics, v. 47, p. 181-192, 2015. 
HOLLNAGEL, E. Prologue: the scope of resilience engineering. Resilience engineeringin practice: a guidebook, Farnham, UK: Ashgate, p.19-29, 2011.

HOUCHENS, N.; KIM, S. C. The Application of Lean in the Healthcare Sector: Theory and Practical Examples, In Wickramasinghe et al., Lean Thinking for Healthcare, Springer New York, p. 43-53, 2014.

JACOBSON, M. J. et al. Designs for learning about climate change as a complex system. Learning and Instruction, v. 52, p. 1-14, 2017.

JOHNSON, N. Simply complexity: a clear guide to complexity theory. Oxford: OneWorld; 2010.

KAPLAN, G.; PATTERSON S. Seeking perfection in healthcare: A case study in adopting Toyota Production System methods. Healthcare executive, v. 23, pp.16-21, 2008.

KIM, C. S.; et al. Lean health care: what can hospitals learn from a world-class automaker?, Journal of Hospital Medicine. v. 1, n. 3, 191-199, 2006.

KURTZ, C. F.; SNOWDEN, D. J. The new dynamics of strategy: sense-making in acomplex-complicated world. IBM Journal of Research \& Development, v.42, p.46283, 2003.

MANS, R.; W. VAN, D. E. A; VANWERSCH, R. J. Process Mining in Healthcare: Evaluating and Exploiting Operational Healthcare, Processes, 2015.

MARCONI, M. A.; LAKATOS, E. M. Fundamentos da metodologia científica. São Paulo: Altas, 2010.

MIGUEL, C. P. A. Metodologia de Pesquisa em Engenharia de Produção. Rio de Janeiro: Elsevier, 2010.

MINTZBERG, H. Managing the myths of health care, World Hosp. Health Serv. Journal International Hospital Federation, v 48, n. 3, p. 4-7, 2012.

MOLDOVAN, F. New Approaches and Trends in Health Care. Procedia Manufacturing, v. 22, p. 947-951, 2018.

MONTERO, R. Los fundamentos teóricos de la práctica de la gestión de la seguridad bajo escrutinio, en Protección \& Seguridad, v. 59 (350), p. 6-14, 2013.

NARAYANAMURTHY, G.; GURUMURTHY, A. Is the hospital lean? A mathematical model for assessing the implementation of lean thinking in healthcare institutions.

Operations Research for Health Care, v. 18, p. 84-98, 2018.

NARAYANAMURTHY, G.; GURUMURTHY, A. Leanness assessment: a literature review. International Journal of Operations \& Production Management, v. 36 (10), p. 1115-1160, 2016.

OLIVEIRA, M.L.; JORDÃO, C.; VERGARA, L.G.L. Fatores biomecânicos: uma análise do trabalho dos enfermeiros. Saber Humano, v. 9, n. 14, p. 154-166, 2019.

PAVARD, B., DUGDALE, J. A Tutorial on Complexity in Social Sciences. Disponível em: http://www.irit.fr/COSI. Acesso em: 20, set. 2018. 
PINTO, J. P. Pensamento Lean: A filosofia das organizações vencedoras. Lisboa: Lidel, 2009.

POKSINSKA, B. B.; FIALKOWSKA-FILIPEK, M.; ENGSTRÖM, J. Does Lean healthcare improve patient satisfaction? A mixed-method investigation into primary care. BMJ Quality and Safety, v. 26, n. 2, p. 95-103, 2018.

RADNOR, Z. Implementing Lean in Health Care: Making the link between the approach, readiness and sustainability. International Journal of Industrial Engineering and Management, v.2(1), p.1-12, 2011.

RENIERS, G.; SORENSEN, K.; KHAN, F. Amyotte P.Resilience of chemical industrial areas through attenuationbased security. Reliability Engineering \& System Safety, v. 131, p. 94-101, 2014.

RIGHI, A. W.; SAURIN, T. A.; WACHS, P. A. systematic literature review of resilience engineering: Research areas and a research agenda proposal. Reliability Engineering and System Safety, v. 141, p. 142-152, 2015.

ROJAS, E. et al. Process mining in healthcare: A literature review. Journal of Biomedical Informatics, v. 61, p. 224-236, 2016.

SAURIN, T. A. et al. The design of scenario-based training from the resilience engineering perspective: A study with grid electricians. Accident Analysis and Prevention, v. 68, p. 30-41, 2014.

SNOWDEN, D. F.; BOONE, M. E. A leader's framework for decision making: wise executives tailor their approach to fit the complexity of the circumstances they face. Harvard Business Review, p. 69-76, 2007.

SOLIMAN, M.; SAURIN, T. A. Lean production in complex socio-technical systems: A systematic literature review. Journal of Manufacturing Systems, v. 45, p. 135-148, 2017a.

SOLIMAN, M.; SAURIN, T. A. Uma análise das barreiras e dificuldades em lean healthcare. Revista Produção Online, v.17, n. 2, p. 620-640, 2017 b.

SOUZA, B. L.; PIDD, M. Exploring the barriers to lean health care implementation. Public Money \& Management, v. 31 (1), p. 59-66, 2011.

SOUZA, L.; SOUZA. Trends and approaches in lean healthcare. Leadership in health services, v. 22(2), p. 121-139, 2009.

TRIST, E. The evolution of socio-technical systems: a conceptual framework and action research program. In: Occasional Paper No. 2. Ontario, CA: Ontario Quality of Working Life Centre; 2017.

WACHS, P. et al. Resilience skills as emergent phenomena : A study of emergency departments in Brazil and the United States. Applied Ergonomics, v. 56, p. 227-237, 2016.

WALKER, G.H. et al. Translating concepts of complexity to the field of ergonomics. Ergonomics, v. 53, p. 1175-86, 2010. 
WAN, J. et al. How attachment influences users willingness to donate to content creators in social media: A sociotechnical systems perspective. Information \& Management, v. 54, n. 7, p. 837-850, 2017.

WELLMAN, J.; HAGAN, P.; JEFFRIES, H. Leading the Lean Healthcare Journey. New York: Taylor and Francis Group, 2011.

YOUNG, T. et al. Using industrial processes to improve patient care. BMJ, vol. 328, p. 162-164, 2004. 